\title{
Boundary Controllability of a Pseudoparabolic Equation
}

\author{
Qiang Tao, ${ }^{1}$ Hang Gao, ${ }^{2}$ and Zheng-an Yao ${ }^{1}$ \\ ${ }^{1}$ School of Mathematics and Computational Science, Sun Yat-Sen University, Guangzhou 510275, China \\ ${ }^{2}$ School of Mathematics and Statistics, Northeast Normal University, Changchun 130024, China \\ Correspondence should be addressed to Qiang Tao; taoq060@126.com
}

Received 24 May 2013; Revised 29 July 2013; Accepted 30 July 2013

Academic Editor: Valery Y. Glizer

Copyright (C) 2013 Qiang Tao et al. This is an open access article distributed under the Creative Commons Attribution License, which permits unrestricted use, distribution, and reproduction in any medium, provided the original work is properly cited.

We deal with the controllability problem for the pseudoparabolic equation by means of boundary controls. Due to the unusual spectrum of this kind of equations, we prove that the null controllability property is false. Furthermore, by the explicit solution, we show that the approximate controllability holds.

\section{Introduction}

This paper is devoted to the study of controllability properties of the $1 \mathrm{D}$ pseudoparabolic equation:

$$
\begin{gathered}
y_{t}-y_{x x}-k y_{x x t}=0, \quad x \in(0,1), t \in(0, T), \\
y(0, t)=0, \quad y(1, t)=u(t), \quad t \in(0, T), \\
y(x, 0)=y_{0}(x), \quad x \in(0,1),
\end{gathered}
$$

where $y=y(x, t)$ is the state, $u=u(t)$ is a control function acting on the boundary $\{1\} \times(0, T)$, and $k>0$ is a constant.

The pseudoparabolic equations are a kind of SobolevGalpern type equations. They have occurred in numerous physical applications among which include problems involving seepage of fluids through fissured rocks [1], unsteady flows of second-order fluids [2,3], and the theory of thermodynamics involving two temperatures [4]. They can also be used as a regularization of ill-posed transport problems, especially as a quasicontinuous approximation to discrete models for population dynamics [5]. Furthermore, pseudoparabolic equations are closely related to the well-know BBM equations [6] which are advocated as a refinement of $\mathrm{KdV}$ equations.

In the last two decades, important progress has been made in the controllability analysis of parabolic equations. We refer to the works [7-13] and the references therein. It is well known that the null and approximate controllability hold for the classical parabolic equations. However, for some special models, there arise some new results. For example, in [14], the authors considered the heat equation with memory:

$$
y_{t}-\Delta y+\int_{0}^{t} \Delta y(x, s) d s=0
$$

By establishing that the observability inequality for the heat equation with memory is not true, they proved that there exists a set of initial conditions such that the null controllability property fails by means of boundary controls. Recently, Doubova and Fernández-Cara [15] studied the approximate distributed and boundary controllability of viscoelastic fluids of the Jeffreys kind, which can be equivalently rewritten as a parabolic equation with memory

$$
y_{t}-\nu \Delta y-b \int_{0}^{t} e^{-a(t-s)} \Delta y(s) d s+\nabla \pi=e^{-a t} \nabla \cdot \tau_{0} .
$$

The main tool for proving the approximate controllability result is a unique continuation property for its adjoint system. The authors pointed out that this parabolic equation with memory transforms from a damped wave equation (see [15]):

$$
z_{t t}-a z_{t}-v \Delta z_{t}-b \Delta z+\nabla\left(e^{a t} \pi\right)=\nabla \cdot \tau_{0} .
$$

In recent years, there are more and more works addressing the controllability problems of damped wave equations (see e.g., [16-19] and the references cited therein).

In this paper, we focus on another kind of parabolic equation with damped term $y_{t x x}$, that is, so-called the 
pseudoparabolic equation. Inspired by the above works, whether the pseudoparabolic equation is controllable or not seems very interesting. Indeed, as the third-order term arises, some properties of (1) are quite different from those of the parabolic equations. One of the most essential differences is that, comparing with the fact that the eigenvalues of heat equations accumulate at $-\infty$, the eigenvalues of (1) have an accumulation real point $-1 / k$ (it will be shown in Section 2). A similar property was pointed in [20]. This difference causes that the controllability properties of (1) become deeply different from the ones for the parabolic equations. We will show that system (1) is not null controllable under the influence of such unusual spectrum. To this end, we turn the control problem into a moment problem. Thanks to the Paley-Wiener theorem, the result is got, and our approach avoids the proof of the observability inequalities which was used in [14]. On the other hand, we establish the approximate controllability of system (1) in some Fourier definition of Sobolev spaces $H_{\alpha}$. The proof is based on a duality method and the explicit solution of the adjoint system of (1), which we obtain by variable separations instead of the Laplace transform in [15]. The techniques we use to prove the approximate controllability contain some ideas of the unique continuation properties.

Throughout this paper, we will use the following notations. The $n$th Fourier coefficient (with respect to the orthonormal basis $(\sin (n \pi x))_{n \geq 1}$ of $\left.L^{2}(0,1)\right)$ of any integrable function $y:(0,1) \rightarrow \mathbb{R}$ is defined as

$$
\widehat{y}_{n}=\int_{0}^{1} y(x) \sin (n \pi x) d x .
$$

So, $y$ can be written in the form

$$
y(x)=\sum_{n \geq 1}\left(\widehat{y}_{n}\right) \sin (n \pi x) .
$$

For any $\alpha \in \mathbb{R}$, let

$$
H_{\alpha}:=\left\{y:(0,1) \longrightarrow \mathbb{R} ; \sum_{n \geq 1} n^{2 \alpha}\left|\widehat{y}_{n}\right|^{2}<\infty\right\} .
$$

Endowed with the scalar product

$$
(y, z)_{\alpha}=\sum_{n \geq 1} n^{2 \alpha} \widehat{y}_{n} \widehat{z}_{n}
$$

$H_{\alpha}$ is a Hilbert space. Moreover, $H_{1}=H_{0}^{1}(0,1), H_{2}=H^{2}(0$, 1) $\bigcap H_{0}^{1}(0,1)$, and $H_{-\alpha}=H_{\alpha}^{\prime}$ (the dual space of $H_{\alpha}$ with respect to the central space $\left.H_{0}=L^{2}(0,1)\right)$ for any $\alpha \geq 0$. Finally, for any $f=\sum_{n \geq 1} c_{n} \sin (n \pi x) \in H_{-\alpha}$ and any $g=$ $\sum_{n \geq 1} d_{n} \sin (n \pi x) \in H_{\alpha}$, we have that

$$
\langle f, g\rangle_{H_{-\alpha}, H_{\alpha}}=\sum_{n \geq 1} c_{n} d_{n}
$$

where $\langle\cdot, \cdot\rangle_{H_{-\alpha}, H_{\alpha}}$ stands for the duality pairing between $H_{-\alpha}$ and $H_{\alpha}$.

The main aim of this paper is to analyze the controllability properties of (1). It will be said that (1) is approximate controllable by boundary control at time $T$; if for any $y_{0} \in$ $H_{0}^{1}(0,1)$, the set of reachable states

$$
\begin{aligned}
R(T)=\{ & y(T)=y(T ; u): \\
& \left.y \text { is the solution of }(1) \text { with } u \in H_{0}^{1}(0, T)\right\}
\end{aligned}
$$

is dense in $H_{\alpha}$. And it will be said that (1) is null controllable at time $T$; if for any given $y_{0} \in H_{0}^{1}(0,1)$, there exist controls $u \in H_{0}^{1}(0, T)$ such that the associated solutions to (1) satisfy

$$
y(x, T)=0 \quad \text { in } \Omega .
$$

For the sake of simplicity, we will take $k=1$ throughout this paper. All the results can be extended without difficulty to $k>0$ arbitrary.

The rest of this paper is organized as follows. In Section 2, we will show some elementary properties for (1) and their adjoint equation. Section 3 is devoted to studying the null and approximate controllability of (1), respectively. In Section 4, some open questions related to this work are provided.

\section{Preliminaries}

In this section, we first consider the existence and uniqueness of the solution to problem (1).

Let $y(x, t)=z(x, t)+x u(t)$. We readily obtain that $z$ is a solution of the system

$$
\begin{gathered}
z_{t}-z_{x x}-z_{x x t}=-x u^{\prime}, \quad x \in(0,1), \quad t \in(0, T), \\
z(0, t)=z(1, t)=0, \quad t \in(0, T), \\
z(x, 0)=y_{0}(x)-x u(0), \quad x \in(0,1) .
\end{gathered}
$$

The existence and uniqueness of the solution to the problem (12) are well known (see $[21,22]$ ); that is, for every $y_{0} \in$ $H_{0}^{1}(0,1)$ and $u \in H_{0}^{1}(0, T)$, system (12) admits a unique solution $z \in C\left([0, T] ; L^{2}(0,1)\right)$. In turn, we see that if $y_{0} \in$ $H_{0}^{1}(0,1)$ and $u \in H_{0}^{1}(0, T)$, system (1) admits a unique solution $y \in C\left([0, T] ; L^{2}(0,1)\right)$.

In order to prove the controllability of system (1), let us consider the following homogeneous initial boundary value problem:

$$
\begin{gathered}
y_{t}-y_{x x}-y_{x x t}=0, \quad x \in(0,1), t \in(0, T), \\
y(0, t)=y(1, t)=0, \quad t \in(0, T), \\
y(x, 0)=y_{0}(x), \quad x \in(0,1) .
\end{gathered}
$$

We will give an explicit solution of the problem (13) by the method of separation of variables.

Proposition 1. If the initial condition $y_{0}$ is given by $y_{0}(x)=$ $\sum_{n \geq 1} a_{n} \sin (n \pi x)$, then the solution to (13) is

$$
y(x, t)=\sum_{n \geq 1} a_{n} e^{\lambda_{n} t} \sin (n \pi x),
$$


where $\lambda_{n}=\mu_{n} /\left(1-\mu_{n}\right)$ and $\mu_{n}=-(n \pi)^{2}$. Moreover, $\lambda_{n}<0$, $\left\{\lambda_{n}\right\}_{n \geq 1}$ is a monotone decreasing sequence, and $\lambda_{n} \rightarrow-1$ as $n \rightarrow \infty$.

Proof. Let $y(x, t)=T(t) X(x) \neq 0$. From the first equality of (13), we have

$$
\frac{T^{\prime}(t)}{T(t)+T^{\prime}(t)} \equiv \frac{X^{\prime \prime}(x)}{X(x)} .
$$

We can see that the identity is true if and only if both sides of it are equal to one constant. Let the constant be $\mu$. Then we get

$$
\begin{gathered}
X^{\prime \prime}(x)=\mu X(x), \quad 0<x<1, \\
T^{\prime}(t)-\mu T(t)-\mu T^{\prime}(t)=0, \quad t>0 .
\end{gathered}
$$

Since the solution $y(x, t)$ satisfies the boundary condition, $X(0)=X(1)=0$ is necessary. Thus, we obtain an eigenvalue problem

$$
\begin{gathered}
X^{\prime \prime}(x)=\mu X(x), \quad 0<x<1, \\
X(0)=X(1)=0 .
\end{gathered}
$$

By using a simple calculation, we have that $\mu=\mu_{n}=-(n \pi)^{2}$ and

$$
X_{n}(x)=B_{0} \sin (n \pi x),
$$

where $B_{0}$ is an arbitrary constant. Now, let us turn to the second equation of (16)

$$
T^{\prime}(t)-\mu_{n} T^{\prime}(t)-\mu_{n} T(t)=0, \quad t>0 .
$$

It is easy to see that

$$
T_{n}(t)=B_{1} e^{\lambda_{n} t}
$$

is the solution to (19), where $\lambda_{n}=\mu_{n} /\left(1-\mu_{n}\right)$ and $B_{1}$ is an arbitrary constant. Combining the initial condition $y_{0}$, we can write the solution of (13) as

$$
y(x, t)=\sum_{n \geq 1} a_{n} e^{\lambda_{n} t} \sin (n \pi x) .
$$

The expressions of $\lambda_{n}$ imply that $\lambda_{n}<0,\left\{\lambda_{n}\right\}_{n \geq 1}$ is a monotone decreasing sequence, and $\lambda_{n} \rightarrow-1$ as $n \rightarrow \infty$.

Remark 2. It is important to observe that the spectrum of (1) is quite different from that of heat equation. This will be essential when dealing with the controllability problem of (1).

As an easy consequence of the above representation formula, we have the following result.

Proposition 3. Let $\alpha \in \mathbb{R}$. If $y_{0} \in H_{\alpha}$, then $y \in C\left(\mathbb{R}^{+} ; H_{\alpha}\right)$. In addition, if $\alpha>3 / 2$, then $\sum_{n \geq 1} n\left|a_{n}\right|<+\infty$ and $y_{x}(1, \cdot) \in$ $C\left(\mathbb{R}^{+}\right)$.
Proof. If $y_{0} \in H_{\alpha}$, we have $\sum_{n \geq 1} n^{2 \alpha}\left|a_{n}\right|^{2}<+\infty$. It holds that

$$
n^{2 \alpha}\left|a_{n} e^{\lambda_{n} t}\right|^{2} \leq n^{2 \alpha}\left|a_{n}\right|^{2} e^{2 \lambda_{n} t} \leq n^{2 \alpha}\left|a_{n}\right|^{2},
$$

and hence, $y \in C\left(\mathbb{R}^{+} ; H_{\alpha}\right)$.

On the other hand, taking the derivative with respect to $x$ in (14), we obtain that

$$
y_{x}(1, t)=\sum_{n \geq 1} a_{n} e^{\lambda_{n} t}(n \pi)(-1)^{n} .
$$

Since

$$
\begin{aligned}
\sum_{n \geq 1} n\left|a_{n} e^{\lambda_{n} t}\right| & \leq \sum_{n \geq 1} n\left|a_{n}\right| \\
& \leq\left(\sum_{n \geq 1} n^{2 \alpha}\left|a_{n}\right|^{2}\right)^{1 / 2} \cdot\left(\sum_{n \geq 1} n^{-2(\alpha-1)}\right)^{1 / 2},
\end{aligned}
$$

we see that $\sum_{n \geq 1} n\left|a_{n}\right|<+\infty$ and $y_{x}(1, \cdot) \in C\left(\mathbb{R}^{+}\right)$, provided that $\alpha>3 / 2$.

Now, let us turn to the adjoint system to system (1) as follows:

$$
\begin{gathered}
\varphi_{t}+\varphi_{x x}-\varphi_{x x t}=0, \quad x \in(0,1), t \in(0, T), \\
\varphi(0, t)=\varphi(1, t)=0, \quad t \in(0, T), \\
\varphi(x, T)=\varphi^{T}(x), \quad x \in(0,1) .
\end{gathered}
$$

Based on the method in Proposition 1, we have that if $\varphi^{T}$ is decomposed as

$$
\varphi^{T}(x)=\sum_{n \geq 1} b_{n} \sin (n \pi x),
$$

the solution of (25) is given by

$$
\varphi(x, t)=\sum_{n \geq 1} b_{n} e^{\lambda_{n}(T-t)} \sin (n \pi x),
$$

which yields

$$
\varphi_{x}(1, t)=\sum_{n \geq 1} b_{n} e^{\lambda_{n}(T-t)}(n \pi)(-1)^{n} .
$$

Corresponding to Proposition 3, we have the following result.

Proposition 4. Let $\alpha \in \mathbb{R}$. If $\varphi^{T} \in H_{-\alpha}$, then $\varphi \in C\left(\mathbb{R}^{+} ; H_{-\alpha}\right)$. In addition, if $\alpha<-3 / 2$, then $\sum_{n \geq 1} n\left|b_{n}\right|<+\infty$ and $\varphi_{x}(1, \cdot) \in$ $C\left(\mathbb{R}^{+}\right)$.

Proof. If $\varphi^{T} \in H_{-\alpha}$, we have $\sum_{n \geq 1} n^{-2 \alpha}\left|b_{n}\right|^{2}<\infty$. It follows that

$$
n^{-2 \alpha}\left|b_{n} e^{\lambda_{n}(T-t)}\right|^{2} \leq n^{-2 \alpha}\left|b_{n}\right|^{2} .
$$

Hence $\varphi \in C\left(\mathbb{R}^{+} ; H_{-\alpha}\right)$. On the other hand, we have by the Cauchy-Schwarz inequality that

$$
\sum_{n \geq 1} n\left|b_{n}\right| \leq\left(\sum_{n \geq 1} n^{2 \alpha+2}\right)^{1 / 2} \cdot\left(\sum_{n \geq 1} n^{-2 \alpha}\left(\left|b_{n}\right|^{2}\right)\right)^{1 / 2} .
$$

It is clear that $\sum_{n \geq 1} n\left|b_{n}\right|<+\infty$, provided that $\alpha<-3 / 2$. 


\section{The Main Results}

This section is devoted to the study of the controllability of system (1). We first do some transformation by the duality principle.

Multiplying (formally) the first equation in (1) by $\varphi$ which is the solution of $(25)$ and integrating on $(0,1) \times(0, T)$, we have

$$
\int_{0}^{T} \int_{0}^{1} y_{t} \varphi d x d t-\int_{0}^{T} \int_{0}^{1} y_{x x} \varphi d x d t-\int_{0}^{T} \int_{0}^{1} y_{x x t} \varphi d x d t=0
$$

Integrating by parts, we get

$$
\begin{aligned}
\int_{0}^{1} y(x, T) \varphi(x, T) d x-\int_{0}^{T} \int_{0}^{1} y \varphi_{t} d x d t \\
\quad+\int_{0}^{T} y(1, t) \varphi_{x}(1, t) d t-\int_{0}^{T} \int_{0}^{1} y \varphi_{x x} d x d t \\
\quad+\int_{0}^{T} y_{t}(1, t) \varphi_{x}(1, t) d t \\
\quad-\int_{0}^{1} y(x, T) \varphi_{x x}(x, T) d x \\
\quad+\int_{0}^{T} \int_{0}^{1} y \varphi_{x x t} d x d t \\
\quad+\int_{0}^{1} y_{0}(x)\left(\varphi_{x x}(x, 0)-\varphi(x, 0)\right) d x=0 .
\end{aligned}
$$

Noticing the first equation in (25), we obtain

$$
\begin{aligned}
& \int_{0}^{1} y(x, T)\left(\varphi_{x x}(x, T)-\varphi(x, T)\right) d x \\
& \quad-\int_{0}^{1} y_{0}(x)\left(\varphi_{x x}(x, 0)-\varphi(x, 0)\right) d x \\
& \quad=\int_{0}^{T} y_{t}(1, t) \varphi_{x}(1, t) d t+\int_{0}^{T} y(1, t) \varphi_{x}(1, t) d t .
\end{aligned}
$$

It is clear that (33) can be rewritten as

$$
-\left.\left\langle\varphi-\varphi_{x x}, y\right\rangle_{H_{-\alpha}, H_{\alpha}}\right|_{0} ^{T}=\int_{0}^{T}\left(u(t)+u^{\prime}(t)\right) \varphi_{x}(1, t) d t .
$$

Now, we are ready to show the controllability of system (1).

3.1. Null Controllability. Following some of the key ideas developed by Micu [23], we are able to show that the null controllability property fails.

Theorem 5. For $T>0$, there exist initial conditions $y_{0} \in$ $H_{0}^{1}(0,1)$ such that for any control function $u \in H_{0}^{1}(0, T)$, the associated solution $y$ to system (1) is not identically equal zero at time $T$.
Proof. It follows from (34) that the null controllability problem is equivalent to the existence of a control function $u=$ $u(t)$ such that

$$
\begin{aligned}
\left\langle\varphi(x, 0)-\varphi_{x x}(x, 0), y_{0}(x)\right\rangle_{H_{-\alpha}, H_{\alpha}} \\
=\int_{0}^{T}\left(u(t)+u^{\prime}(t)\right) \varphi_{x}(1, t) d t .
\end{aligned}
$$

Using (9), (27), and (28), we can transform the control problem into a moments problem. In other words, we need to find the control function $u=u(t)$ such that

$$
\int_{0}^{T}\left(u(t)+u^{\prime}(t)\right) e^{-\lambda_{n} t}(n \pi)(-1)^{n} d t=a_{n}\left(1+n^{2} \pi^{2}\right) .
$$

To this end, we consider that any initial data $y_{0}$ with the sequence $\left\{a_{n}\right\}_{n \geq 1}$ satisfies $a_{n}=0$ for $n>N$. Suppose that for this kind of $y_{0}$, there exists a $u$ such that (36) holds. Let

$$
F(z):=\int_{0}^{T}\left(u(t)+u^{\prime}(t)\right) e^{i z t} d t
$$

By Paley-Wiener theorem, $F$ is an entire function and

$$
F\left(i \lambda_{n}\right)=\int_{0}^{T}\left(u(t)+u^{\prime}(t)\right) e^{-\lambda_{n} t} d t=0, \quad \text { for } n>N
$$

The fact that $i \lambda_{n} \rightarrow-i$ as $n \rightarrow+\infty$ implies that $F$ is zero on a set with a finite accumulation point. Therefore, $F \equiv 0$. It follows that $a_{n}=0$ for each $n \geq 1$. Thus the proof of Theorem 5 is completed.

3.2. Approximate Controllability. Because of the lack of null controllability, the approximate controllability of system (1) becomes much more interesting. We will study the approximate controllability in this part. Without loss of generality, we assume that $y_{0}=0$ and by (34) we have

$$
\begin{array}{r}
-\left\langle\varphi^{T}(x)-\varphi_{x x}^{T}(x), y(x, T)\right\rangle_{H_{-\alpha}, H_{\alpha}} \\
=\int_{0}^{T}\left(u(t)+u^{\prime}(t)\right) \varphi_{x}(1, t) d t .
\end{array}
$$

In order to show the approximate controllability, two lemmas are needed later. The first one is an equivalent condition for the approximate controllability.

Lemma 6. For system (25), if we assume that $\varphi^{T}=0$ if and only if $\int_{0}^{T}\left(u(t)+u^{\prime}(t)\right) \varphi_{x}(1, t) d t=0$ for any function $u=u(t)$, then system (1) is approximatively controllable in $H_{\alpha}$.

Proof. Recall that the approximate controllability of system (1) in $H_{\alpha}$ is equivalent to that $R(T)$ that is dense in $H_{\alpha}$. Therefore, in order to conclude the proof, we only need to show that $R(T)$ is dense in $H_{\alpha}$. It follows from Hahn-Banach theorem that every continuous linear functional on $H_{\alpha}$ which vanishes on $R(T)$, must vanish everywhere on $H_{\alpha}$. 
Now, suppose that $R(T)$ is not dense in $H_{\alpha}$. Then by Hahn-Banach theorem there exists $\varphi^{T} \in H_{-\alpha}$ with $\varphi^{T}-$ $\varphi_{x x}^{T} \neq 0$, such that for any $y(x, T) \in R(T)$, we have

$$
\left\langle\varphi^{T}(x)-\varphi_{x x}^{T}(x), y(x, T)\right\rangle_{H_{-\alpha}, H_{\alpha}}=0 .
$$

By (39), we get that

$$
\int_{0}^{T}\left(u(t)+u^{\prime}(t)\right) \varphi_{x}(1, t) d t=0 .
$$

But from the condition $\varphi^{T}=0$ if and only if $\int_{0}^{T}(u(t)+$ $\left.u^{\prime}(t)\right) \varphi_{x}(1, t) d t=0$ for any function $u=u(t)$, we have that 0 is the only initial value $\varphi^{T} \in H_{-\alpha}$ for which the solution $\varphi(x, t)$ of $(25)$ fulfills $\int_{0}^{T}\left(u(t)+u^{\prime}(t)\right) \varphi_{x}(1, t) d t=0$ for any function $u=u(t)$. Therefore $\varphi^{T}=0$, and it is contrary to the choice of $\varphi^{T}$. This completes the proof of Lemma 6 .

Lemma 6 implies that, in order to prove the approximate controllability of system (1) with boundary control, we only need to check the condition about the solution of the dual problem (25) in Lemma 6.

The following elementary lemma can be found in $[24,25]$.

Lemma 7. Let $\left(\beta_{n}\right)_{n>1}$ and $\left(\lambda_{n}\right)_{n>1}$ be two sequences of complex numbers such that $\sum_{n \geq 1}\left|\beta_{n}\right|<\infty$ and $\operatorname{Re} \lambda_{n}<\Lambda$ for each $n \geq 1$ and some number $\Lambda \in \mathbb{R}$. Assume that the $\lambda_{n}$ s are pairwise distinct and that $\sum_{n \geq 1} \beta_{n} e^{\lambda_{n} t}=0$ for a.e. $t \in(0, T)$. Then $\beta_{n}=0$ for all $n \geq 1$.

We are now in a position to present the proof of the approximate controllability of (1) with boundary control.

Theorem 8. Let $T>0$. System (1) is approximately controllable in $H_{\alpha}$ with $\alpha<-3 / 2$ at time $T$.

Proof. First, we prove that there is a control function $u$ such that $R(T)$ is dense in $H_{\alpha}$ with $\alpha<-3 / 2$. Now, take $\varphi^{T} \in H_{-\alpha}$ with $\alpha<-3 / 2$ decomposed as in (26).

Set $\theta=u+u^{\prime}$ and denote

$$
G:=\left\{g \in L^{2}(0, T) ; \int_{0}^{T} e^{t} g(t) d t=0\right\} .
$$

Then it follows that when $u$ ranges over $H_{0}^{1}(0, T)$ and $\theta$ ranges over $G$.

Assume that, for any $u \in H_{0}^{1}(0, T)$,

$$
\int_{0}^{T} \theta(t) \varphi_{x}(1, t) d t=\int_{0}^{T}\left(u(t)+u^{\prime}(t)\right) \varphi_{x}(1, t) d t=0 .
$$

By Lemma 6, we only need to prove that $\varphi^{T}=0$ or equivalently that $b_{n}=0$ for each $n$ (see (26)). Noticing that for any $\theta \in \operatorname{Span}\left(e^{t}\right)^{\perp}$,

$$
\left(\theta, \varphi_{x}(1, \cdot)\right)_{L^{2}(0, T)}=0 .
$$

We have $\varphi_{x}(1, \cdot) \in \operatorname{Span}\left(e^{t}\right)^{\perp \perp}=\operatorname{Span}\left(e^{t}\right)$. Therefore, from (28), there exists a constant $C \in \mathbb{R}$ such that

$$
\sum_{n \geq 1} b_{n} e^{\lambda_{n}(T-t)}(n \pi)(-1)^{n}=C e^{t},
$$

for a.e. $t \in(0, T)$. If taking $\tau=T-t, c_{n}=b_{n}(n \pi)(-1)^{n}$, and $c_{0}:=-C e^{T}$, we obtain

$$
\sum_{n \geq 1} c_{n} e^{\lambda_{n} \tau}+c_{0} e^{-\tau}=0,
$$

for a.e. $\tau \in(0, T)$.

On the other hand, since $\varphi^{T} \in H_{-\alpha}$ with $\alpha<-3 / 2$ and by Proposition 4, we have that

$$
\sum_{n \geq 1}\left|c_{n}\right|=\pi \sum_{n \geq 1} n\left|b_{n}\right|<+\infty .
$$

Then by Lemma 7, we obtain that $c_{n}=0$ for each $n \geq 1$. It implies that $\varphi^{T}=0$. The proof of Theorem 8 is completed.

\section{Concluding Remark}

In this paper, the controllability of the pseudoparabolic equation is studied. With a boundary control, it is proved that the system is not null controllable, but that an approximate controllability result is obtained in some appropriate functional spaces. Below is a list of unsolved and interesting questions related to our work.

(1) It has been got that the approximate controllability holds in $H_{\alpha}$ with $\alpha<-3 / 2$. The question whether the approximate controllability holds for $\alpha \geq-3 / 2$ remains open. The method in this paper does not work for that case.

(2) It seems natural to expect that the controllability for multidimensional pseudoparabolic equations through a boundary controller or a locally distributed one. We will consider these problems in the forthcoming papers.

(3) It would be quite interesting to study the controllability properties of (1) for the case variable coefficient (i.e., $k=k(x, t)$ ). However, it seems very difficult, and many classical methods such as moment problem and strongly continuous semigroups may be false. Indeed, controllability of equation with variable coefficients always bring us much more difficult than that with constant coefficient. One needs a highly innovative way to obtain the observability inequalities or unique continuation properties. An example was presented in [26] to establish some controllability results for wave equations with variable coefficients by a Riemannian geometry method.

\section{Acknowledgment}

The authors would like to thank the anonymous reviewer for their valuable comments, which significantly contributed 
to improving the quality of the paper. This work was supported by the NSF of China under Grants 11171060, 11271381, 11301345, and 11071036, National Basic Research Program of China (973 Program) (no. 2011CB808002).

\section{References}

[1] G. I. Barenblatt, I. P. Zheltov, and I. N. Kochina, "Basic concepts in the theory of seepage of homogeneous liquids in fissured rocks," Journal of Applied Mathematics and Mechanics, vol. 24, pp. 1286-1303, 1960.

[2] B. D. Coleman, R. J. Duffin, and V. J. Mizel, "Instability, uniqueness and nonexistence theorems for the equation $u_{t}=$ $u_{x x}-u_{x t x}$ on a strip," Archive for Rational Mechanics and Analysis, vol. 19, pp. 100-116, 1965.

[3] T. W. Ting, "Certain non-steady flows of second-order fluids," Archive for Rational Mechanics and Analysis, vol. 14, pp. 1-26, 1963.

[4] P. J. Chen and M. E. Gurtin, "On a theory of heat conduction involving two temperatures," Zeitschrift für Angewandte Mathematik und Physik, vol. 19, pp. 614-627, 1968.

[5] V. Padrón, "Effect of aggregation on population revovery modeled by a forward-backward pseudoparabolic equation," Transactions of the American Mathematical Society, vol. 356, no. 7, pp. 2739-2756, 2004.

[6] T. B. Benjamin, J. L. Bona, and J. J. Mahony, "Model equations for long waves in nonlinear dispersive systems," Philosophical Transactions of the Royal Society of London A, vol. 272, no. 1220, pp. 47-78, 1972.

[7] V. Barbu, "Controllability of parabolic and Navier-Stokes equations," Scientiae Mathematicae Japonicae, vol. 56, no. 1, pp. 143211, 2002.

[8] A. Doubova, E. Fernández-Cara, M. González-Burgos, and E. Zuazua, "On the controllability of parabolic systems with a nonlinear term involving the state and the gradient," SIAM Journal on Control and Optimization, vol. 41, no. 3, pp. 798-819, 2002.

[9] E. Fernández-Cara and E. Zuazua, "Null and approximate controllability for weakly blowing up semilinear heat equations," Annales de l'Institut Henri Poincaré. Analyse Non Linéaire, vol. 17, no. 5, pp. 583-616, 2000.

[10] C. Fabre, J.-P. Puel, and E. Zuazua, "Approximate controllability of the semilinear heat equation," Proceedings of the Royal Society of Edinburgh A, vol. 125, no. 1, pp. 31-61, 1995.

[11] A. V. Fursikov and O. Yu. Imanuvilov, Controllability of Evolution Equations, Seoul University, Seoul, Republic of Korea, 1996.

[12] X. Zhang, "A remark on null exact controllability of the heat equation," SIAM Journal on Control and Optimization, vol. 40, no. 1, pp. 39-53, 2001.

[13] G. Wang, " $L^{\infty}$-null controllability for the heat equation and its consequences for the time optimal control problem," SIAM Journal on Control and Optimization, vol. 47, no. 4, pp. 17011720, 2008.

[14] S. Guerrero and O. Y. Imanuvilov, "Remarks on non controllability of the heat equation with memory," ESAIM. Control, Optimisation and Calculus of Variations, vol. 19, no. 1, pp. 288300, 2013.

[15] A. Doubova and E. Fernández-Cara, "On the control of viscoelastic Jeffreys fluids," Systems \& Control Letters, vol. 61, no. 4, pp. 573-579, 2012.
[16] J. L. Boldrini, A. Doubova, E. Fernández-Cara, and M. González-Burgos, "Some controllability results for linear viscoelastic fluids," SIAM Journal on Control and Optimization, vol. 50, no. 2, pp. 900-924, 2012.

[17] M. A. Shubov, "Exact boundary and distributed controllability of radial damped wave equation," Journal de Mathématiques Pures et Appliquées, vol. 77, no. 5, pp. 415-437, 1998.

[18] A. López, X. Zhang, and E. Zuazua, "Null controllability of the heat equation as singular limit of the exact controllability of dissipative wave equations," Journal de Mathématiques Pures et Appliquées. Neuvième Série, vol. 79, no. 8, pp. 741-808, 2000.

[19] F. W. Chaves-Silva, L. Rosier, and E. Zuazua, "Null controllability of a system of viscoelasticity with a moving control," Journal de Mathématiques Pures et Appliquées, 2013.

[20] A. Münch and E. Zuazua, "Numerical approximation of null controls for the heat equation: Ill-posedness and remedies," Inverse Problems, vol. 26, no. 8, Article ID 085018, 39 pages, 2010.

[21] R. E. Showalter, "Partial differential equations of SobolevGalpern type," Pacific Journal of Mathematics, vol. 31, pp. 787793, 1969.

[22] R. E. Showalter and T. W. Ting, "Pseudoparabolic partial differential equations," SIAM Journal on Mathematical Analysis, vol. 1, pp. 1-26, 1970.

[23] S. Micu, "On the controllability of the linearized BenjaminBona-Mahony equation," SIAM Journal on Control and Optimization, vol. 39, no. 6, pp. 1677-1696, 2001.

[24] Q. Tao, H. Gao, and B. Zhang, "Approximate controllability of a parabolic equation with memory," Nonlinear Analysis. Hybrid Systems, vol. 6, no. 2, pp. 839-845, 2012.

[25] L. Rosier and P. Rouchon, "On the controllability of a wave equation with structural damping," International Journal of Tomography \& Statistics, vol. 5, pp. 79-84, 2007.

[26] P.-F. Yao, "On the observability inequalities for exact controllability of wave equations with variable coefficients," SIAM Journal on Control and Optimization, vol. 37, no. 5, pp. 15681599, 1999. 


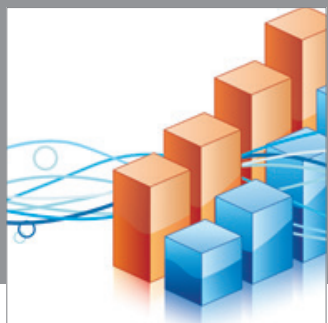

Advances in

Operations Research

mansans

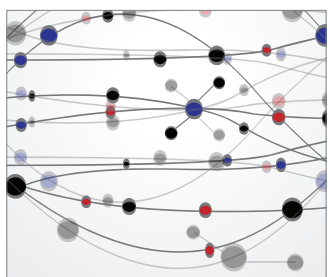

The Scientific World Journal
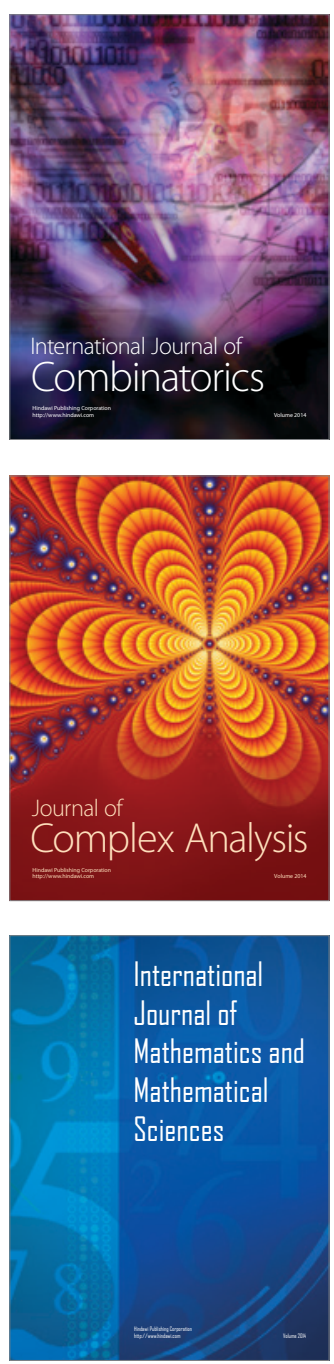
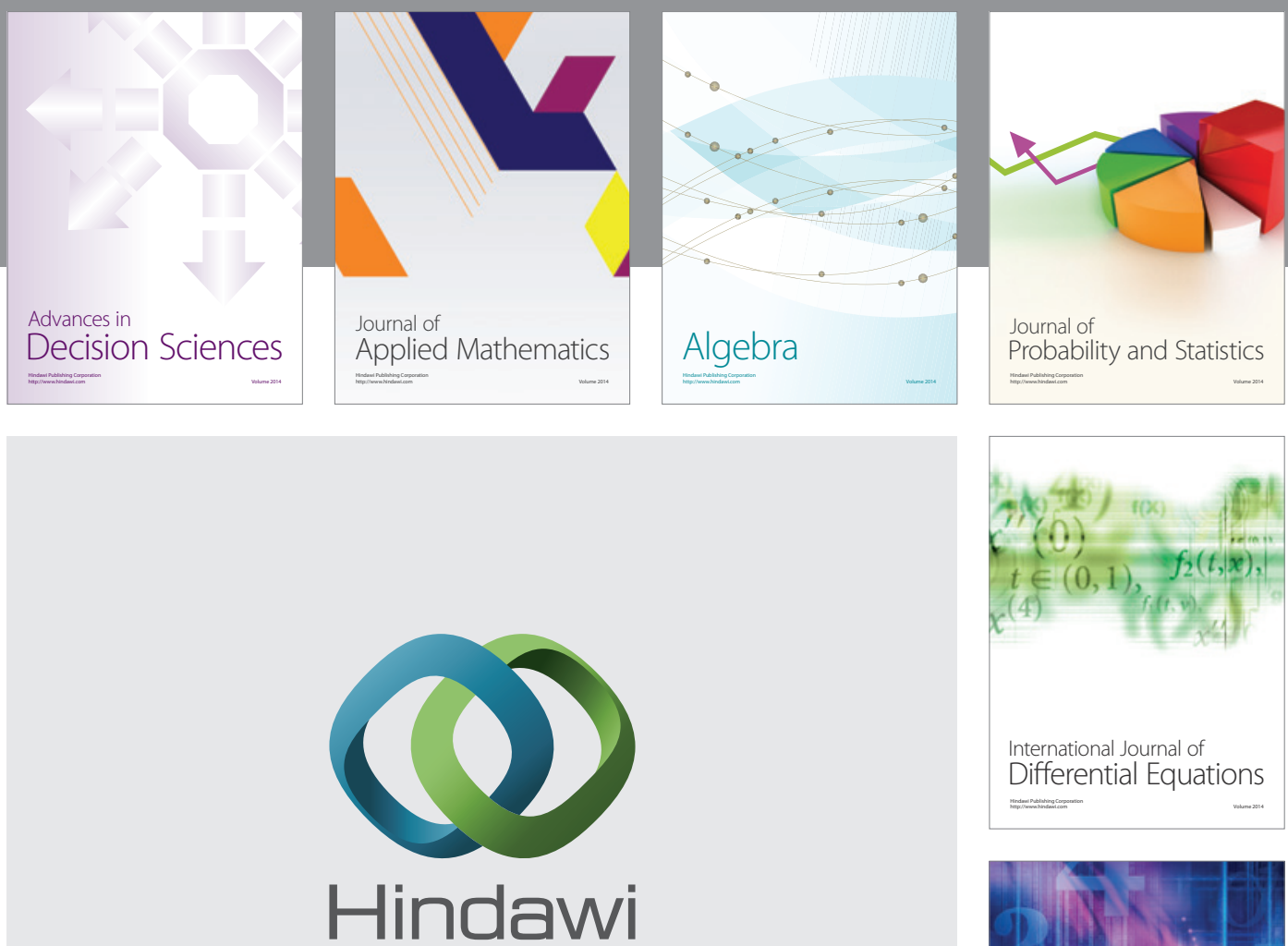

Submit your manuscripts at http://www.hindawi.com
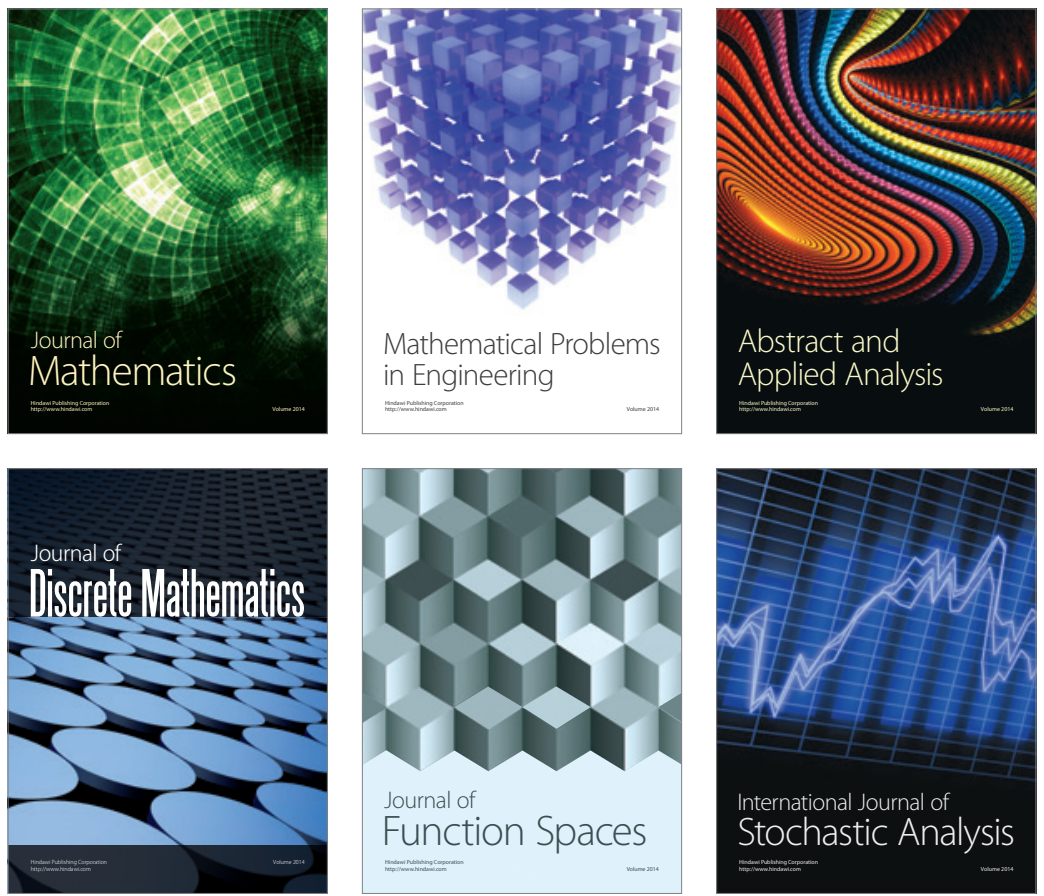

Journal of

Function Spaces

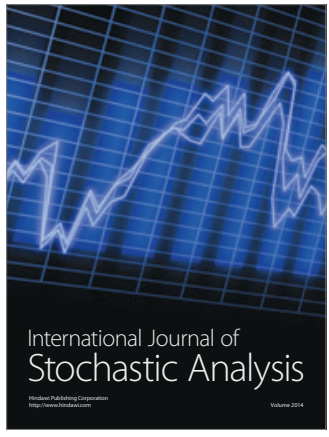

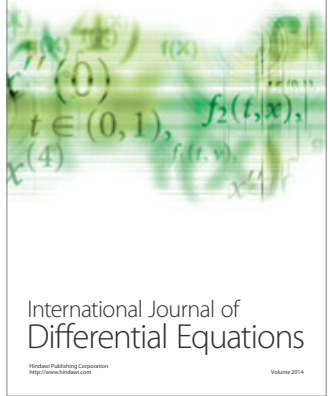
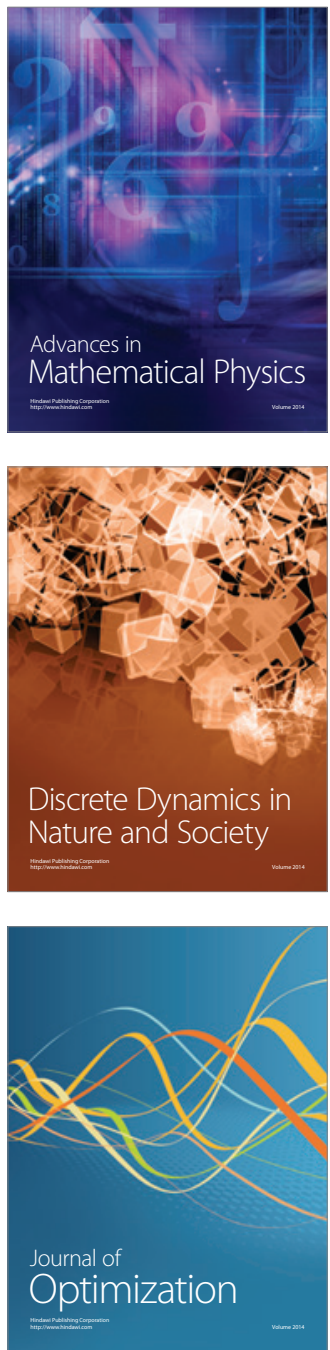